\title{
The association between FTO rs9939609 gene polymorphism and anthropometric indices in adults
}

\author{
Mahsa Mehrdad ${ }^{1}$, Majid Fardaei ${ }^{2}$, Mohammad Fararouei ${ }^{3}$ and Mohammad Hassan Eftekhari ${ }^{{ }^{*}}$ (D
}

\begin{abstract}
Background: Fat mass and obesity-associated gene (FTO) is the most studied obesity-related gene up to date. We aimed to assess anthropometric indices in carriers of FTO rs9939609 polymorphism with overweight across Iranian population (Shiraz) to find out the associations of this polymorphism with obesity indices.

Methods: This was a cross-sectional study conducted on 198 overweight healthy adults aged 20-45 years old. We assessed the body composition of the participants using bioelectrical impedance analyzer. In addition, we measured the waist circumference (WC) and hip circumference $(\mathrm{HC})$. Waist to hip ratio (WHR) and waist to height ratio (WHtR) were also calculated by equations. The participants' genotype was determined by ARMS-PCR. Also, data analysis was performed using SPSS software version 20 and R software version 3.6.2.

Results: The mean values of body mass index (BMI) and age of the participants were $26.93 \pm 1.13 \mathrm{~kg} / \mathrm{m}^{2}$ and 33.33 \pm 6.35 years old, respectively. Homozygous carriers of A-allele had significantly higher values for BMI $\left(0.60 \mathrm{~kg} / \mathrm{m}^{2}, p\right.$ $=0.026)$, WHR (0.04 unit, $p=0.003)$, and WHtR (0.02 unit, $p=0.030)$ than the homozygous carriers of T-allele. Individuals with AA genotype had greater WC $(2.66 \mathrm{~cm}, p=0.042$, and $4.03 \mathrm{~cm}, p=0.002)$, fat mass $(2.24 \mathrm{~kg}, p=$ 0.004 , and $3.02 \mathrm{~kg}, p=0.001)$, and trunk fat $(1.53 \mathrm{~kg}, p=0.001$, and $2.08 \mathrm{~kg}, p=0.001)$ compared to those with AT and $\Pi$ genotypes, respectively. Interestingly, after adjustment of the confounders, significant associations were observed among rs9939609 polymorphism and BMI, Wt, WC, trunk fat percentage, WHR, and WHtR.

Conclusions: A-allele of the FTO rs9939609 polymorphism was indicated to be associated with greater general and central obesity in adult population of Shiraz, Iran.
\end{abstract}

Keywords: Adiposity, Genotype, Overweight, Body mass index, Body fat distribution

\section{Background}

Obesity is a growing critical concern worldwide. The interactions between environmental and genetic factors are known as the main leading causes of obesity [1]. Among obesity-associated genetic factors, the fat mass and obesity-associated (FTO) gene on chromosome 16 has been recognized as one of the most studied genes to date.

\footnotetext{
*Correspondence: h_eftekhari@yahoo.com

'Department of Clinical Nutrition, School of Nutrition \& Food Sciences, Shiraz University of Medical Sciences, Shiraz, Iran

Full list of author information is available at the end of the article
}

The FTO gene encodes a 2-oxoglutarate-dependent nucleic acid demethylase, which catalyzes the demethylation of 3-methylthymine in single-stranded DNA [2]. Although the FTO gene is expressed in most tissues, the highest expression level of this gene is in the hypothalamus [3]. Also, a recent study revealed a new function for $F T O$ as a $\mathrm{Fe}^{2+}$. sensitive transcriptional repressor, which indicates its own ability to form an auto-regulatory loop that may be linked with the hypothalamic control of the body weight [4]. The strongest effect on obesity was found for rs9939609 polymorphism $(\mathrm{A} / \mathrm{T})$ variant in intron 1 [3]. 
Scuteri et al. [5] reported that several SNPs in FTO were associated with body mass index (BMI), total body weight, and also with hip circumference $(\mathrm{HC})$. Interestingly, Zhang et al. [6] observed that several FTO SNPs including rs9939609 polymorphism can affect the body fatness, fat-free mass (FFM), frame size, and muscle mass. Rauhio et al. [7] reported that rs 9939609 polymorphism is associated with body weight but not with body composition and fat distribution in premenopausal women. They observed that the individuals with AA genotype had a higher weight $(\sim 3.6 \mathrm{~kg}$ and $\sim 10.1 \mathrm{~kg}$, respectively) compared to those with AT and TT genotypes, and also, the carriers of A-allele had higher BMI and fat mass (FM) than non-carriers. Andreasen et al. [8] in a large sample of Danes found that the homozygous carriers of the A-allele had a higher weight than non-carriers, which is reflected in $1.1 \mathrm{~kg} / \mathrm{m}^{2}$ for BMI and $2.3 \mathrm{~cm}$ for WC. As shown in the abovementioned studies, there are inconsistent results on the associations and effect sizes of rs 9939609 polymorphism related to obesity indices across different populations and the FTO gene polymorphisms are associated with obesity through various mechanisms [9].

According to the abovementioned studies and the discrepancies, there is a need to replicate such studies in different geographical regions to determine the effect size of this SNP in all ethnic groups [10].

Since there are limited studies conducted in various regions of Iran, we aimed to investigate the association among FTO rs9939609 polymorphism and anthropometric indices, body composition, and fat distribution in healthy overweight adults in Shiraz, Iran.

\section{Methods}

\section{Participants}

We recruited 198 overall healthy overweight adults at Shohadaye WalFajr Health Center in Shiraz, Iran. All the participants were Persian, one of the ethnicities in Iran that is considered as a Caucasian subpopulation. The inclusion criteria were the age range between 20 and 45 years old and BMI $\geq 25.0$ and $\leq 29.9$; not participating in any weight management programs in the last 2 months; no weight loss greater than $5 \%$ in the last 2 months; non-emigrants; non-smokers; not using alcohol or any medications; no specific diseases such as neurological or psychiatric disorders, thyroid, liver, renal, and infectious diseases, and any other specific conditions or disorders; and also not being pregnant or lactating. All participants signed the informed consent form before participating in the study.

\section{Anthropometric assessment}

We measured the height (barefoot) in a standardized measuring position by a non-stretch tape at the first visit. Anthropometric indices were assessed without wearing shoes and while wearing light cloths using Bioelectric Impedance Analyzer (BIA) (Tanita, Japan/BC418). To ensure that the measurements are as precise as possible, we asked the participants to come with an empty stomach and empty bladder, consume no alcohol or large volume of other beverages within $24 \mathrm{~h}$ before the test, and also avoid from doing exercise for $4 \mathrm{~h}$ before the test. We picked out the following markers from the results of BIA assessment: weight, BMI, FM, FM percentage, FFM, trunk fat (TF), TF percentage, and total body water (TBW). We measured the waist circumference (WC) at the midpoint between the lower rib and the iliac crest using a non-stretch tape. $\mathrm{HC}$ was measured at the level of maximum extension of the hip using a non-stretch tape. We calculated WHR using equation of $\mathrm{WC}_{(\mathrm{cm})} / \mathrm{HC}_{(\mathrm{cm})}$ and $\mathrm{WHtR}$ by the equation of $\mathrm{WC}_{(\mathrm{cm})} / \mathrm{Ht}_{(\mathrm{cm})}$.

\section{Genotyping}

Two milliliter of the peripheral venous blood samples was collected into EDTA tubes. The blood samples were stored at $-70^{\circ} \mathrm{C}$ until use. Genomic DNA was extracted by DNA isolation kit (SinaPure DNA Kit, PR881612/ EX6001/CinnaGen/Iran) according to the manufacturer's recommendations. The DNA quantity was measured NanoDrop (ND1000, USA), and the extracted DNA was stored at $-20^{\circ} \mathrm{C}$ until use. We determined the genotypes for FTO rs9939609 polymorphism (TT/ AT/AA) via amplification refractory mutation systempolymerase chain reaction (ARMS-PCR). The method consists of two PCRs, which in one of them, the 3' terminus of the reverse primer is specific for the normal allele, and in the other one, $3^{\prime}$ terminus is specific for the mutated allele. The sequence of the forward primer is GTGAGGAATACTAGGAGAGGAGAA, and the sequences of the reverse primers are AGAGACTATC CAAGTGCATCAGA for the normal T-allele and CAGAGACTATCCAAGTGCATCAAT for the mutated A-allele. All amplification reactions were performed in the ABI Thermocycler Veriti in a volume of $20 \mu \mathrm{l}$ containing $10 \mu \mathrm{l}$ of Mastermix, $1.8 \mu \mathrm{l}$ extracted DNA, $6.8 \mu \mathrm{l}$ of distilled water, and $0.7 \mu \mathrm{l}$ of each forward and reverse primers. The thermal step program used the following compromised stages of one 15 -min cycle at $95{ }^{\circ} \mathrm{C}$ (denaturation), 35 cycles under the following conditions: denaturation at $95^{\circ} \mathrm{C}$ for $30 \mathrm{~s}$, annealing at $56^{\circ} \mathrm{C}$ for $30 \mathrm{~s}$, extension at $72{ }^{\circ} \mathrm{C}$ for $30 \mathrm{~s}$, and a final extension of $72{ }^{\circ} \mathrm{C}$ for $7 \mathrm{~min}$ after performing PCR; $5 \mu \mathrm{l}$ of the product was mixed by $2 \mu \mathrm{l}$ loading dye and then examined by electrophoresis on a $2.5 \%$ agarose gel. When a 351-bp band was obtained in both reactions, the subject from whom DNA was extracted was heterozygous (AT). When the band was obtained in only one of the two PCRs, the 
subject was homozygous. The homozygosity was TT if the band was obtained with the reverse primer with an A-residue in the $3^{\prime}$ position and AA if the T-residue was present in the $3^{\prime}$ position of the reverse primer.

\section{DNA sequencing}

In order to validate the results obtained from the ARMS-PCR method, direct sequencing of the region containing the mutation was performed for one case of the heterozygote genotype and the two cases of the homozygote genotypes, which were randomly selected. The sequencing primers were above forward primer and ACAAATGTTCAAGTCACACTCAG as reverse primer.

\section{Statistical analysis}

The normality of the data distribution was examined by Shapiro-Wilk test. The results from descriptive analyses were presented as mean \pm standard deviation (SD) for performing quantitative continuous variables. Frequency tables were used to present the qualitative variables. Also, the significance level was set at 0.05 . The associations of genotype with quantitative variables were tested using one-way ANOVA test. The Tukey post hoc test was performed to find any significant differences among the compared groups. The allele frequencies were estimated from the genotype information using the direct gene counting method. These statistical analyses were performed using the SPSS software (version 20). In addition, multiple linear regression was applied to assess the adjusted associations between polymorphism alleles and anthropometric indices by adjusting potentially important confounders (of gender, age, educational level, and marital status). The model-fitting procedures and checking for the normality of the residuals were conducted using $R$ version 3.6.2. Since we were blinded to the individuals' genotype at the visit session, no bias was expected for selecting the participants.

\section{Results}

Regarding the FTO rs9939609 genotype, about half of the participants were AT $(n=99), 31 \%$ of them were TT $(n=60)$, and approximately $19 \%$ of them were homozygote for the known risk allele $(n=39)$. Minor allele frequency (MAF) was 44.7\%. The observed genotype frequencies were not significantly different from what was predicted under the assumption of Hardy-Weinberg equilibrium. Details of the participants' characteristics are shown in Table 1.

Table 2 shows the mean and standard deviations for the variables that were divided into three genotype groups. ANOVA test showed the differences in the variables among the three genotype groups (Table 2). There

Table 1 Basic characteristics of the study population

\begin{tabular}{|c|c|c|c|c|}
\hline Characteristics & & Men & Women & Total \\
\hline$N(\%)$ & & $50(25 \%)$ & $148(75 \%)$ & 198 \\
\hline Age (years) & & $34.00 \pm 6.17$ & $33.10 \pm 6.41$ & $33.33 \pm 6.35$ \\
\hline \multirow[t]{3}{*}{ Genotype (\%) } & $\mathrm{AA}$ & $10(25.6 \%)$ & $29(74.4 \%)$ & 39 (19.7\%) \\
\hline & AT & $25(25.3 \%)$ & $74(74.7 \%)$ & $99(50 \%)$ \\
\hline & $\pi$ & $15(25.0 \%)$ & $45(75.0 \%)$ & $60(30.3 \%)$ \\
\hline Height (cm) & & $177.16 \pm 6.32$ & $159.99 \pm 5.57$ & $164.32 \pm 9.44$ \\
\hline Weight (kg) & & $85.11 \pm 6.85$ & $68.89 \pm 5.98$ & $72.99 \pm 9.39$ \\
\hline \multicolumn{5}{|l|}{ Anthropometric indices } \\
\hline $\mathrm{BMI}\left(\mathrm{kg} / \mathrm{m}^{2}\right)$ & & $27.09 \pm 1.22$ & $26.87 \pm 1.10$ & $26.93 \pm 1.13$ \\
\hline Waist circumference (cm) & & $98.90 \pm 5.16$ & $94.56 \pm 5.78$ & $95.66 \pm 5.93$ \\
\hline Hip circumference $(\mathrm{cm})$ & & $103.90 \pm 3.73$ & $102.46 \pm 4.87$ & $102.83 \pm 4.65$ \\
\hline Waist to hip ratio (WHR) & & $0.95 \pm 0.05$ & $0.92 \pm 0.06$ & $0.93 \pm 0.06$ \\
\hline Waist to height ratio (WHtR) & & $0.56 \pm 0.03$ & $0.59 \pm 0.03$ & $0.58 \pm 0.04$ \\
\hline Fat mass $(\mathrm{kg})$ & & $19.19 \pm 4.24$ & $23.44 \pm 3.00$ & $22.36 \pm 3.82$ \\
\hline Fat mass percent & & $22.44 \pm 3.94$ & $34.01 \pm 3.18$ & $31.09 \pm 6.07$ \\
\hline Fat-free mass (kg) & & $65.92 \pm 5.18$ & $45.46 \pm 4.51$ & $50.63 \pm 10.06$ \\
\hline Fat-free mass percent & & $77.56 \pm 3.94$ & $65.99 \pm 3.18$ & $68.91 \pm 6.07$ \\
\hline Total body water (kg) & & $47.47 \pm 6.44$ & $30.51 \pm 3.53$ & $34.79 \pm 8.61$ \\
\hline Trunk fat (kg) & & $10.91 \pm 2.60$ & $12.01 \pm 2.91$ & $11.73 \pm 2.27$ \\
\hline Trunk fat percent & & $56.88 \pm 5.32$ & $51.05 \pm 4.30$ & $52.52 \pm 5.22$ \\
\hline
\end{tabular}

Data are presented as mean \pm standard deviation

$N$ (\%) number (frequency) of individuals, BMI body mass index 
Table 2 Comparison of anthropometric parameters across different genotypes of FTO rs9939609 in the study population

\begin{tabular}{|c|c|}
\hline \multirow[t]{2}{*}{ Items } & Genotype (mean \pm SD) \\
\hline & AT $(n=99)$ \\
\hline
\end{tabular}

Total

$\begin{array}{lllll}\text { Age (years) } & 34.03 \pm 5.89 & 32.99 \pm 6.49 & 33.43 \pm 6.46 & 0.683\end{array}$

$\begin{array}{lllll}\text { Height }(\mathrm{cm}) & 165.72 \pm 8.96 & 163.98 \pm 9.41 & 163.98 \pm 9.84 & 0.591\end{array}$

$\begin{array}{lllll}\text { Weight }(\mathrm{kg}) & 75.24 \pm 9.21 & 72.62 \pm 9.17 & 72.14 \pm 9.81 & 0.238\end{array}$

BMI $\left(\mathrm{kg} / \mathrm{m}^{2}\right) \quad 27.31 \pm 1.32^{\mathrm{a}} \quad 26.91 \pm 1.04^{\mathrm{ab}} \quad 26.71 \pm 1.11^{\mathrm{b}} \quad 0.033^{*}$

WC (cm) $\quad 98.21 \pm 6.60^{\mathrm{a}} \quad 95.55 \pm 5.47^{\mathrm{b}} \quad 94.18 \pm 5.74^{\mathrm{c}} \quad 0.004^{*}$

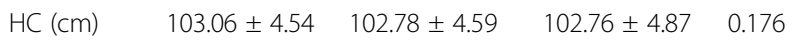

WHR $\quad 0.95 \pm 0.07^{\mathrm{a}} \quad 0.93 \pm 0.05^{\mathrm{ab}} \quad 0.92 \pm 0.05^{\mathrm{b}} \quad 0.005^{*}$

WHtR $\quad 0.593 \pm 0.044^{\mathrm{a}} \quad 0.583 \pm 0.034^{\mathrm{ab}} \quad 0.575 \pm 0.030^{\mathrm{b}} \quad 0.039^{*}$

FM (kg) $\quad 24.40 \pm 4.17^{\mathrm{a}} \quad 22.16 \pm 3.33^{\mathrm{b}} \quad 21.38 \pm 3.91^{\mathrm{c}}<0.001^{*}$

FM (\%) $\quad 32.77 \pm 6.16 \quad 31.04 \pm 5.94 \quad 30.08 \pm 6.08 \quad 0.098$

FFM (kg) $\quad 50.84 \pm 9.55 \quad 50.46 \pm 10.25 \quad 50.76 \pm 10.23 \quad 0.973$

FFM (\%) $\quad 67.23 \pm 6.16 \quad 68.96 \pm 5.94 \quad 69.92 \pm 6.08 \quad 0.098$

TBW (kg) $\quad 35.93 \pm 9.02 \quad 35.02 \pm 8.86 \quad 33.68 \pm 7.92 \quad 0.420$

TF (kg) $\quad 13.13 \pm 2.40^{\mathrm{a}} \quad 11.60 \pm 1.99^{\mathrm{b}} \quad 11.04 \pm 2.27^{\mathrm{c}}<0.001^{*}$

$\begin{array}{lllll}\text { TF }(\%) & 54.09 \pm 5.89 & 52.39 \pm 4.78 & 51.72 \pm 5.34 & 0.082\end{array}$

$\mathrm{a}, \mathrm{b}$, and $\mathrm{c}$ Different values based on the Tukey test

${ }^{\mathrm{ab}}$ The value is not significantly different from others

${ }^{*} p$ value $<0.05$ considered as significant difference between three genotypes (one-way ANOVA results)

were significant differences among the three genotype groups in BMI, WC, WHR, WHtR, FM, and TF. This indicated that FTO rs9939609 polymorphism might affect these variables. The Tukey test showed the two groups had different means, and also, the mean difference was observed for each variable among groups. Significant differences among the three genotype groups were observed for WC, FM, and TF. A significant difference was observed only between the carriers AA and TT for BMI, WHR, and WHtR (Table 3).

Multiple linear regression analysis after adjustment for age, gender, marital status, and educational level showed that this polymorphism was significantly associated with BMI, WC, WHR, and WHtR; however, it was not associated with FM. Though for FM and FFM percentages, the $p$ values were marginal. In addition, the fitted models suggested significant associations of rs9939609 with Wt and TF percentages. The observed associations were significant only for the carriers of AA genotype in comparison to non-carriers. However, the association for WHtR was significant for both carriers of the AA and AT genotypes comparing to TT genotype (Table 4).

\section{Discussion}

We found that the risk allele (A-allele) of FTO rs9939609 polymorphism, particularly in the form of homozygous genotype, was associated with higher BMI,
Table 3 Mean difference of variables among the genotypes

\begin{tabular}{|c|c|c|c|}
\hline Items & Genotypes & Mean difference & $p$ value \\
\hline \multirow[t]{3}{*}{$\mathrm{BMI}\left(\mathrm{kg} / \mathrm{m}^{2}\right)$} & AAwith AT & 0.41 & 0.138 \\
\hline & AA with $\Pi$ & 0.60 & $0.026^{*}$ \\
\hline & AT with $\Pi T$ & 0.20 & 0.526 \\
\hline \multirow[t]{3}{*}{ WC (cm) } & AAwith AT & 2.66 & $0.042^{*}$ \\
\hline & AA with $\Pi$ & 4.03 & $0.002^{*}$ \\
\hline & AT with TT & 1.37 & 0.320 \\
\hline \multirow[t]{3}{*}{ WHR } & AA with AT & 0.02 & 0.052 \\
\hline & AA with $\Pi$ & 0.04 & $0.003^{*}$ \\
\hline & AT with $\Pi$ & 0.01 & 0.306 \\
\hline \multirow[t]{3}{*}{ WHtR } & AA with AT & 0.01 & 0.300 \\
\hline & AA with $\Pi T$ & 0.02 & $0.030^{*}$ \\
\hline & AT with TT & 0.01 & 0.295 \\
\hline \multirow[t]{3}{*}{ FM (kg) } & AA with AT & 2.24 & $0.004^{*}$ \\
\hline & AA with $\Pi$ & 3.02 & $<0.001^{*}$ \\
\hline & AT with TT & 0.78 & 0.401 \\
\hline \multirow[t]{3}{*}{ TF (kg) } & AA with AT & 1.53 & $0.001^{*}$ \\
\hline & AA with $\Pi$ & 2.08 & $<0.001^{*}$ \\
\hline & AT with TT & 0.56 & 0.258 \\
\hline
\end{tabular}

WC, FM, TF, WHR, and WHtR. However, after adjustment for age, gender, marital status, and educational level, these associations remained significant just for BMI, WC, WHR, and WHtR. In addition, this analysis showed that there were also significant associations for Wt and TF percentages. The significant association did not remain significant for FM and TF anymore, after adjusting the confounders. These findings indicate that the risk allele might affect adiposity, specifically central obesity. The small difference observed in the effect size of these polymorphism alleles might result in misinterpretation by clinicians as a low clinical importance. It should be noted that these differences were caused by the effect of one SNP. Several SNPs in combination might lead to greater effects, which clinically makes the outcomes more critical. These findings showed that this polymorphism might affect the body fat distribution in addition to general fatness.

The risk allele frequency observed in this population (44.7\%) was close to the frequency in Europeans (45.0\%) and inconsistent with the frequency of East Asians (12.6\%) [11]. Accordingly, it is an important finding since it reflects the higher frequency of the individuals with risk allele, and subsequently higher frequency of the individuals with the homozygous genotype for risk allele in this population of Iranians. It can be concluded that this polymorphism is a considerable risk factor for 
Table 4 Associations of anthropometric variables and rs9939609 polymorphism

\begin{tabular}{|c|c|c|c|c|}
\hline Variable & Genotype & $B$ coefficient & 95\% confidence interval & $p$ value \\
\hline \multirow[t]{2}{*}{ BMI $\left(\mathrm{kg} / \mathrm{m}^{2}\right)$} & AT & 0.24 & -0.10 to 0.59 & 0.168 \\
\hline & AA & 0.58 & 0.15 to 1.01 & $0.001^{*}$ \\
\hline \multirow[t]{2}{*}{ Weight (kg) } & AT & 0.59 & -1.36 to 2.54 & 0.552 \\
\hline & AA & 2.89 & 0.45 to 5.34 & $0.020^{*}$ \\
\hline \multirow[t]{2}{*}{ Waist circumference $(\mathrm{cm})$} & AT & 1.58 & -0.07 to 3.23 & 0.059 \\
\hline & AA & 3.81 & 1.73 to 5.88 & $<0.001^{*}$ \\
\hline \multirow[t]{2}{*}{ Fat mass (kg) } & AT & 0.89 & 0.89 to 0.51 & 0.083 \\
\hline & $A A$ & 2.99 & 2.99 to 0.64 & 0.066 \\
\hline \multirow[t]{2}{*}{ Fat mass percentage (\%) } & AT & 1.04 & -0.02 to 2.09 & 0.053 \\
\hline & $A A$ & 2.73 & 1.41 to 4.05 & 0.051 \\
\hline \multirow[t]{2}{*}{ FFM (kg) } & AT & -0.30 & -0.30 to 0.77 & 0.700 \\
\hline & AA & -0.10 & -0.10 to 0.97 & 0.918 \\
\hline \multirow[t]{2}{*}{ FFM percentage (\%) } & AT & -1.04 & -2.09 to 0.02 & 0.053 \\
\hline & AA & -2.73 & -4.05 to -1.41 & 0.051 \\
\hline \multirow[t]{2}{*}{ Trunk fat (kg) } & AT & 0.62 & -0.04 to 1.28 & 0.066 \\
\hline & $A A$ & 2.07 & 1.23 to 2.91 & 0.060 \\
\hline \multirow[t]{2}{*}{ Trunk fat percentage (\%) } & AT & 0.71 & -0.75 to 2.17 & 0.337 \\
\hline & $A A$ & 2.35 & 0.52 to 4.18 & $0.012^{*}$ \\
\hline \multirow[t]{2}{*}{ WHR } & AT & 0.01 & 0.00 to 0.03 & 0.076 \\
\hline & $A A$ & 0.04 & 0.01 to 0.06 & $<0.001^{*}$ \\
\hline \multirow[t]{2}{*}{ WHtR } & AT & 0.01 & 0.00 to 0.02 & $0.048^{*}$ \\
\hline & $A A$ & 0.02 & 0.01 to 0.03 & $0.005^{*}$ \\
\hline
\end{tabular}

Adjusted for gender, age, marital status, and educational level using multiple linear regression

${ }^{*} p$ value $<0.05$ considered as significant

obesity and its comorbidities among the Iranian population, and more research should be conducted in this field, and the clinicians should consider its effects on the prevention and treatment.

There are several pathways suggested for the exact underlying mechanism of the effect of FTO on obesity. Remarkably, FTO is the first recognized demethylase, which reverses $\mathrm{N}^{6}$-methyladenosine $\left(\mathrm{m}^{6} \mathrm{~A}\right)$ in RNA methylation [9]. It has been proposed that $\mathrm{m}^{6} \mathrm{~A}$ regulates adipogenesis through mediating the mRNA splicing [12]. Another study suggested that FTO can regulate adipogenesis through modulation of mitotic clonal expansion [13]. Recently, Wu et al. [14] found that FTO promoted adipogenesis of 3 T3-L1 pre-adipocytes through regulating the cell cycle procession in an $\mathrm{m}^{6} \mathrm{~A}-\mathrm{YTHDF} 2$ dependent way. Tews et al. [15] observed that FTO deficiency induces browning of the white adipose tissue including enhanced UCP-1 expression and mitochondrial uncoupling. Also, they claimed that FTO can raise the susceptibility to obesity and overweight by inhibiting white adipose tissue browning [16]. Also, another study found that FTO gene expression may be associated with a change in the percentage of skeletal muscle [17]. Despite all these suggestions, the functional role of the FTO gene and its variants is not fully understood yet.

In accordance with our findings, Frayling et al. demonstrated that the homozygous carriers of A-allele of rs9939609 polymorphism are the significant risk factors for obesity as they weighed $3 \mathrm{~kg}$ heavier than the homozygotes for the T-allele [3]. Macekova et al. [18] revealed that in a population of the Roma/Gypsy individuals, WC was larger in the participants with AA genotype compared to those with TT genotype. They also observed a strong association between A-allele and BMI. Ghafarian-Alipour et al. [19] observed that women who carried the risk allele of rs9939609 polymorphism had a significantly higher weight and WC than non-carriers. Fawwad et al. [17] observed the increased central obesity with A-allele in Karachi, Pakistan, and Vasan et al. [20] observed that WHR was greater among adolescents with AA genotype than TT genotype. Despite the differences in effect size and age groups, these studies showed that, in line with our study, this variant could affect the body fat distribution and is associated with central obesity in addition to general obesity.

Inconsistent with our results, Rauhio et al. [7] in their study conducted on obese women observed that 
rs9939609 polymorphism was not associated with fat distribution and might only affect general obesity. In their study, differences between the genotypes for WC and $\mathrm{HC}$ were not statistically significant; however, these differences were clinically significant, since we observed that the mean WC in their study for AA genotype was approximately 3 and $7 \mathrm{~cm}$ greater than for AT and TT genotypes, respectively. These differences are greater than those we observed for WC and are also clinically important for one SNP. Interestingly, in another study performed in Tabriz by Majdi et al. [21], no significant difference was found for BMI among different genotypes. It might be due to the different ethnicities of Tabriz and Shiraz, despite belonging to one country (Iran), since the major ethnicity in Tabriz is Turkish and that in Shiraz is Persian, considered as a subpopulation of Caucasians. Likewise, Solak et al. [22] observed no correlations between rs9939609 polymorphism and obesityrelated parameters in the population of Turkey whose ethnicity was Turkish. Their findings were different from ours in the genotype frequencies, MAF, and the impact on obesity as BMI. In their study, MAF of $11.11 \%$ was observed in the healthy individuals, with a frequency of 79.0, 19.7, and $0.4 \%$ for TT, AT, and AA genotypes, respectively. The reason for the discrepancy might be due to the differences among the geographical regions, which might affect the impact of the polymorphisms [22]. In addition, it should be noted that sample size can play a role in the differences observed in the outcomes of various studies.

The frequency that we observed was near the MAF; Mojaver et al. reported for the healthy individuals (42\%) in Isfahan. The frequencies of TT, AT, and AA genotypes were 32,52 , and $16 \%$ in their populations, respectively [23]. This small difference in the frequencies between their study and ours might be due to the geographical variations, since they recruited the individuals from Isfahan and our participants were from Shiraz. These differences necessitate the replication of the polymorphism studies across different geographic regions.

The strength of our study was the recruitment of individuals with a narrow range of BMI $(26.93 \pm 1.13 \mathrm{~kg} /$ $\mathrm{m}^{2}$ ). Therefore, we could not attribute the differences of WC, WHR, WHtR, and TF percentages to being overweight. Further, we could attribute these differences to the type of alleles an individual carries. Another strength of our study was recruiting one ethnicity in the city, which might result in approximate homogeneity for the genetic make-up of the participants, and subsequently valid results.

We recommend the replication of these types of studies across different regions of Iran, and also with other variants of FTO to better understand the effect of this gene on obesity and its management.

\section{Conclusions}

We observed that the risk allele of FTO rs9939609 was associated with greater central and general obesity indices in adults with overweight in Shiraz. This SNP might affect the body fat distribution in addition to general fatness in this population. Further studies are needed to discover the true mechanisms underlying the effects of these genetic variants on obesity.

\section{Abbreviations}

BIA: Bioelectric Impedance Analyzer; BMI: Body mass index; Wt: Weight; WC: Waist circumference; HC: Hip circumference; WHR: Waist to hip ratio; WHtR: Waist to height ratio

\section{Acknowledgements}

This study was conducted at the School of Nutrition and Food Sciences and Shohadaye Walfajr Health Center of Shiraz University of Medical Sciences, Shiraz, Iran. We acknowledge the staff of the mentioned centers for their kind cooperation.

This study was also conducted at the Department of Public Health Nutrition of the Shiraz University of Medical Sciences, Tehran, Iran (Code: 2842). We acknowledge the staff at the participating schools for their excellent cooperation.

\section{Authors' contributions}

MM, MHE, and MF designed the study and were involved in the data collection, analysis, and drafting of the manuscript. MM and MF were involved in the design of the study, analysis of the data, and critically reviewed the manuscript. All authors read and approved the final manuscript.

\section{Funding \\ Funding for this study was provided by Shiraz University of Medical Sciences.}

\section{Availability of data and materials}

Not applicable

\section{Ethics approval and consent to participate}

This study is a cross-sectional study approved by the local ethics review boards at Shiraz University of Medical Sciences (IR.SUMS.REC.1395.100). The study was conducted after obtaining written informed consent from all study participants.

This study has been approved by local ethics review boards at Shiraz University of Medical Sciences (ir.sums.rec.1395.100).

Consent for publication

Institutional consent forms were used in this study.

\section{Competing interests}

The authors declare that they have no competing interests.

\section{Author details}

${ }^{1}$ Department of Clinical Nutrition, School of Nutrition \& Food Sciences, Shiraz University of Medical Sciences, Shiraz, Iran. ${ }^{2}$ Department of Medical Genetic, School of Medicine, Shiraz University of Medical Sciences, Shiraz, Iran. ${ }^{3}$ HIV/ AIDs Research Center, Shiraz University of Medical Sciences, Shiraz, Iran.

Received: 4 November 2019 Accepted: 22 April 2020

Published online: 12 May 2020

\section{References}

1. Maes HH, Neale MC, Eaves LJ. Genetic and environmental factors in relative body weight and human adiposity. Behav Genet. 1997;27(4):325-51.

2. Gerken T, Girard CA, Tung Y-CL, Webby CJ, Saudek V, Hewitson KS, et al. The obesity-associated FTO gene encodes a 2-oxoglutarate-dependent nucleic acid demethylase. Science (New York, NY). 2007;318(5855):1469-72.

3. Frayling TM, Timpson NJ, Weedon MN, Zeggini E, Freathy RM, Lindgren CM, et al. A common variant in the FTO gene is associated with body mass 
index and predisposes to childhood and adult obesity. Science (New York, NY). 2007:316(5826):889-94

4. Liu SJ, Tang HL, He Q, Lu P, Fu T, Xu XL, et al. FTO is a transcriptional repressor to auto-regulate its own gene and potentially associated with homeostasis of body weight. J Mol Cell Biol. 2018.

5. Scuteri A, Sanna S, Chen WM, Uda M, Albai G, Strait J, et al. Genome-wide association scan shows genetic variants in the FTO gene are associated with obesity-related traits. PLoS Genet. 2007;3(7):e115.

6. Zhang G, Karns R, Narancic NS, Sun G, Cheng H, Missoni S, et al. Common SNPs in FTO gene are associated with obesity related anthropometric traits in an island population from the eastern Adriatic coast of Croatia. PLoS One. 2010;5(4):e10375.

7. Rauhio A, Uusi-Rasi K, Nikkari ST, Kannus P, Sievanen H, Kunnas T. Association of the FTO and ADRB2 genes with body composition and fat distribution in obese women. Maturitas. 2013;76(2):165-71.

8. Andreasen CH, Stender-Petersen KL, Mogensen MS, Torekov SS, Wegner L, Andersen $\mathrm{G}$, et al. Low physical activity accentuates the effect of the FTO rs9939609 polymorphism on body fat accumulation. Diabetes. 2008;57(1): 95-101.

9. Jia G, Fu Y, Zhao X, Dai Q, Zheng G, Yang Y, et al. N6-methyladenosine in nuclear RNA is a major substrate of the obesity-associated FTO. Nat Chem Biol. 2011;7(12):885-7.

10. Peng S, Zhu Y, Xu F, Ren X, Li X, Lai M. FTO gene polymorphisms and obesity risk: a meta-analysis. BMC Med. 2011;9:71.

11. Lee HJ, Kim IK, Kang JH, Ahn Y, Han BG, Lee JY, et al. Effects of common FTO gene variants associated with BMI on dietary intake and physical activity in Koreans. Clin Chim Acta. 2010;411(21-22):1716-22.

12. Zhao $X$, Yang $Y$, Sun BF, Shi $Y$, Yang $X$, Xiao W, et al. FTO-dependent demethylation of N6-methyladenosine regulates mRNA splicing and is required for adipogenesis. Cell Res. 2014;24(12):1403-19.

13. Merkestein M, Laber S, McMurray F, Andrew D, Sachse G, Sanderson J, et al. FTO influences adipogenesis by regulating mitotic clonal expansion. Nat Commun. 2015;6:6792

14. Wu R, Liu Y, Yao Y, Zhao Y, Bi Z, Jiang Q, et al. FTO regulates adipogenesis by controlling cell cycle progression via m(6)A-YTHDF2 dependent mechanism. Biochim Biophys Acta Mol Cell Biol Lipids. 2018;1863(10):132330.

15. Tews $\mathrm{D}$, Fischer-Posovszky $P$, Fromme T, Klingenspor M, Fischer J, Ruther $U$, et al. FTO deficiency induces UCP-1 expression and mitochondrial uncoupling in adipocytes. Endocrinology. 2013;154(9):3141-51.

16. Heaton JM. The distribution of brown adipose tissue in the human. J Anat. 1972;112(Pt 1):35.

17. Fawwad A, Siddiqui IA, Zeeshan NF, Shahid SM, Basit A. Association of SNP rs9939609 in FTO gene with metabolic syndrome in type 2 diabetic subjects, recruited from a tertiary care unit of Karachi, Pakistan. Pakistan J Med Sci. 2015;31(1):140-5.

18. Macekova S, Bernasovsky I, Gabrikova D, Bozikova A, Bernasovska J, Boronova I, et al. Association of the FTO rs9939609 polymorphism with obesity in Roma/gypsy population. Am J Phys Anthropol. 2012;147(1):30-4.

19. Ghafarian-Alipour F, Ziaee S, Ashoori MR, Zakeri MS, Boroumand MA, Aghamohammadzadeh N, et al. Association between FTO gene polymorphisms and type 2 diabetes mellitus, serum levels of apelin and androgen hormones among Iranian obese women. Gene. 2018;641:361-6.

20. Vasan SK, Fall T, Job V, Gu HF, Ingelsson E, Brismar K, et al. A common variant in the FTO locus is associated with waist-hip ratio in Indian adolescents. Pediatr Obes. 2013;8(3):e45-9.

21. Majdi MA, Mohammadzadeh NA, Lotfi H, Mahmoudi R, Alipour FG, Shool F, et al. Correlation of resistin serum level with fat mass and obesity-associated gene (FTO) rs9939609 polymorphism in obese women with type 2 diabetes. Diab Metab Syndrome. 2017;11(Suppl 2):S715-s20.

22. Solak M, Ozdemir Erdogan M, Yildiz SH, Ucok K, Yuksel S, Arikan Terzi ES, et al. Association of obesity with rs 1421085 and rs9939609 polymorphisms of FTO gene. Mol Biol Rep. 2014;41(11):7381-6.

23. Mojaver M, Mokarian F, Kazemi M, Salehi M. Specific TaqMan allelic discrimination assay for rs 1477196 and rs9939609 single nucleotide polymorphisms of FTO gene demonstrated that there is no association between these SNPs and risk of breast cancer in Iranian women. Adv Biomed Res. 2015;4:136.

\section{Publisher's Note}

Springer Nature remains neutral with regard to jurisdictional claims in published maps and institutional affiliations.

Ready to submit your research? Choose BMC and benefit from:

- fast, convenient online submission

- thorough peer review by experienced researchers in your field

- rapid publication on acceptance

- support for research data, including large and complex data types

- gold Open Access which fosters wider collaboration and increased citations

- maximum visibility for your research: over $100 \mathrm{M}$ website views per year

At BMC, research is always in progress.

Learn more biomedcentral.com/submissions 